\title{
Postoperative Euglycemic Ketoacidosis in Type 2 Diabetes Associated with Sodium-Glucose Cotransporter 2 Inhibitor: Insights Into Pathogenesis and Management Strategy
}

\author{
Harshith P. Chandrakumar ${ }^{1}$, Seeta Chillumuntala ${ }^{1}$, Gurcharan Singh ${ }^{1}$, Samy I. McFarlane ${ }^{1}$ \\ 1. Department of Medicine, State University of New York (SUNY) Downstate, Health Science University, Brooklyn, USA
}

Corresponding author: Samy I. McFarlane, samy.mcfarlane@downstate.edu

\begin{abstract}
Euglycemic diabetic ketoacidosis (eu-DKA) is an uncommon and serious adverse event associated with the use of sodium-glucose cotransporter (SGLT-2) inhibitors. It is a state of increased anion gap metabolic acidosis with ketosis but in the setting of normal serum glucose levels. Diagnosis of this serious entity could easily be missed given the non-specific symptoms and the normal glucose measurements.

This ketogenic state can be triggered by various stressors including infection, surgery, myocardial infarctions, omission of insulin dosage, as well as low carbohydrate diet. In this report, we present a case of eu-DKA in a 68 -year-old woman with type 2 diabetes that occurred in the postoperative period of glaucoma surgery. She was started shortly before surgery on SGLT-2 inhibitor (ertugliflozin). While the diagnosis was initially missed, it was subsequently confirmed when she presented with reduced appetite, generalized fatigue, and constipation. Ertugliflozin was discontinued, and she was successfully treated with conservative management and without insulin drip.
\end{abstract}

This case highlights the need to consider the diagnosis of eu-DKA in patients treated with SGLT-2 inhibitors since the diagnosis could easily be missed especially in the postoperative period with the non-characteristic symptomatology and normoglycemia.

Review began 05/27/2021 Review ended 05/31/2021 Published 06/08/2021

\section{(๑) Copyright 2021}

Chandrakumar et al. This is an open access article distributed under the terms of the Creative Commons Attribution License CC-BY 4.0., which permits unrestricted use, distribution, and reproduction in any medium, provided the original author and source are credited.
Categories: Endocrinology/Diabetes/Metabolism

Keywords: sodium-glucose cotransporter 2 (sglt-2) inhibitors, ertugliflozin, euglycemic diabetic ketoacidosis, postoperative period, adverse drug reaction, diabetes type 2

\section{Introduction}

Sodium-glucose cotransporter (SGLT-2) inhibitors that are a relatively newer class of antidiabetic medications have emerged as important therapeutic agents that reduce cardiovascular events, hospitalization from heart failure, as well as the progression of chronic kidney disease (CKD) in both diabetic and non-diabetic populations. The therapeutic indications for these agents are rapidly changing the guidelines of the American Diabetes Association (ADA), American College of Cardiology (ACC), as well as other major national and international organizations [1-6]. This class of anti-diabetic drugs specifically acts on the proximal renal tubules, causing a reduction in serum blood glucose through glycosuria [2]. One of the serious adverse effects of SGLT-2 inhibitors is the occurrence of euglycemic diabetic ketoacidosis (euDKA) with an incidence of $\leqslant 0.3 \%$ from recent trials [6-8]. To date, there are four Food and Drug Administration (FDA)-approved SGLT-2 inhibitor drugs that have been approved for type 2 diabetes mellitus, which include canagliflozin, dapagliflozin, empagliflozin, and ertugliflozin. Ertugliflozin is a much newer agent with around 20 cases of eu-DKA reported in the literature, including the Vertis CV trial [8-11]. Of note also, these agents have been increasingly used off-label in type 1 diabetes with multiple reports on eu-DKA in this susceptible population [9].

In this report, we present a case of a 68-year-old woman with type 2 diabetes who developed eu-DKA after initiation of ertugliflozin therapy and was precipitated by recent eye surgery. Of importance, the diagnosis was missed during the initial visit given the non-specific nature of the symptoms and the normal glucose values obtained during the post-operative period. This patient was treated conservatively without insulin drip, indicating that she has insulin secretory capacity and corollary to the management of alcoholic ketoacidosis, which is another sub-type of euglycemic ketoacidosis. We also provide a literature review on the diagnosis and preventive measures of this potentially serious adverse effect of SGLT- 2 inhibitors.

\section{Case Presentation}

\section{Initial admission}

The patient is a 68-year-old woman with hypertension, type 2 diabetes, and recent right eye surgery for 


\section{Cureus}

glaucoma two days prior to admission when she presented with a right-sided headache with blurry vision and slowed speech lasting for a few hours. The symptoms spontaneously resolved during the initial emergency room interview. There was no weakness, incontinence, syncopal episodes, or trauma. Physical exam showed a fixed dilated pupil with decreased visual acuity likely secondary to surgery with no focal neurological deficits. She was admitted for workup of transient ischemic attack.

Of note, she was initiated on ertugliflozin right before the surgery. Initial labs (Table 1) showed an anion gap (AG) of $27 \mathrm{mmol} / \mathrm{L}$ and bicarbonate (HCO3-) of $8 \mathrm{mmol} / \mathrm{L}$ with a blood glucose of $147 \mathrm{mg} / \mathrm{dl}$. A computed tomography (CT) scan of the head, as well as CT angiography of the head and neck, showed no abnormalities. Clinically improved with symptom resolution, she was discharged the next day to continue with home medications. Her medications at the time included simvastatin, metoprolol, and ertugliflozin 15 mg once daily.

\begin{tabular}{|c|c|c|c|}
\hline Labs & Initial admission & Repeat admission & On discharge \\
\hline Hemoglobin (g/dL) & 14.4 & 14.1 & 12.3 \\
\hline White blood cells (K/uL) & 8.16 & 10.02 & 6.61 \\
\hline Platelets (K/uL) & 271 & 197 & 203 \\
\hline Sodium (mmol/L) & 131 & 132 & 137 \\
\hline Potassium (mmol/L) & 4.9 & 4.3 & 3.9 \\
\hline Chloride (mmol/L) & 100 & 94 & 101 \\
\hline CO2 (mmol/L) & 8 & 15 & 21 \\
\hline Blood urea nitrogen(mg/dL) & 19 & 13 & 12 \\
\hline Creatinine(mg/dL) & 1.2 & 0.9 & 0.7 \\
\hline Anion gap(mmol/L) & 27 & 27 & 19 \\
\hline Blood glucose (mg/dL) & 147 & 110 & 151 \\
\hline
\end{tabular}

TABLE 1: Laboratory values during admission and discharge

\section{Repeat admission}

The patient presented eight days later with decreased appetite, generalized malaise, and constipation. The physical exam was unremarkable. However, labs (Table 1) showed a persistently elevated AG of 23, HCO3- of 15 , beta-hydroxybutyrate(BHB) of 4.89 , and $\mathrm{pH}$ of 7.36 on blood gas measurement, together with a lactate of $1.2 \mathrm{mmol} / \mathrm{L}$, hemoglobin A1c of $12.8 \%$, and blood glucose of $110 \mathrm{mg} / \mathrm{dl}$. Urinalysis showed glucose $>500$ $\mathrm{mg} / \mathrm{dL}$ and ketones of $80 \mathrm{mg} / \mathrm{dL}$ (Table 2). She had normal kidney function and denied any substance or alcohol use. Electrocardiogram (EKG) at the time showed new T wave inversions in lead V3, but repeat troponins remained negative. CT of the abdomen with intravenous contrast showed a large stool burden in the rectum and sigmoid colon, and a fleet enema was administered. Upon further questioning, the patient reported non-compliance with anti-diabetic agents, including metformin, with ertugliflozin being the first agent she took consistently. 


\section{Cureus}

\begin{tabular}{|l|l|}
\hline Labs & Result \\
\hline Lactate (mmol/L) & 1.2 \\
\hline Hemoglobin A1c (\%) & 12.6 \\
Beta-hydroxy butyrate (mmol/L) & 4.89 \\
\hline Urine pH & 5 \\
\hline Urine glucose (mg/dL) & $>500$ \\
\hline Urine ketones (mg/dL) & 80 \\
\hline Urine specific gravity & 1.021 \\
\hline Urine nitrite & negative \\
Urine leucocyte esterase & negative \\
Venous blood gas: pH & 7.36 \\
\hline pCO2 (mmHg) & 37.7 \\
\hline pO2 (mmHg) & 30.3 \\
\hline HCO3 (mmHg) & 21 \\
\hline
\end{tabular}

\section{TABLE 2: Additional laboratory values}

\section{Management}

The diagnosis of mild eu-DKA secondary to the SGLT-2 inhibitor was made. An endocrinology consult was sought and was advised to stop ertugliflozin and continue supportive management with intravenous fluids and insulin aspart coverage. The patient was also administered 2 liters of Ringer's lactate solution and was continued on $100 \mathrm{~mL} /$ hour continuous infusion. Diet was re-initiated as tolerated. Soon after ertugliflozin was held, the patient's appetite and bowel movements improved. She was observed for symptomatic improvement and on Day 2 of hospitalization, AG improved to 19 and HCO3- to 21. Blood glucose during the hospital course remained $<140 \mathrm{mg} / \mathrm{dL}$ and did not require any additional insulin. On the last day of hospitalization, the patient noted having the 'best meal' in two weeks as opposed to her reduced appetite previously. Upon discharge, ertugliflozin was held; she was initiated on sitagliptin and discharged home with endocrinology follow-up.

\section{Discussion}

SGLT-2 receptors are located in the early S1 segment of the proximal renal tubules as opposed to the SGLT-1 receptors that are located in the S2, S3 segment. Under normal physiology, $80 \%-90 \%$ of the glucose reaching the proximal tubules is absorbed by the SGLT- 2 receptors, and $10-20 \%$ is absorbed by the SGLT- 1 receptors [2]. SGLT-2 receptors are also located in the alpha 2 pancreatic cells [3]. Being a co-transporter, they absorb glucose with every molecule of sodium into the blood passively.

In nondiabetic individuals, the renal threshold for glucosuria is $180 \mathrm{mg} / \mathrm{dL}$, with most of the glucose being reabsorbed in the proximal renal tubules [2]. But in the diabetic population, the renal threshold is increased, partly due to upregulation of SGLT-2 receptors contributing to increased glucose co-transport leading to hyperglycemia [4]. SGLT-2 inhibitor blocks this mechanism causing reduction of hyperglycemia, independent of insulin and thereby avoiding hypoglycemic episodes. This is manifested by increasing glucosuria and improving insulin sensitivity in peripheral tissues [2]. Although these drugs improve the glycemic index, the occurrence of eu-DKA should be considered.

Diabetic ketoacidosis (DKA) includes hyperglycemia (serum glucose $>250 \mathrm{mg} / \mathrm{dL}$ ), acidosis (arterial pH $<7.3$ and bicarbonate $<15 \mathrm{mEq} / \mathrm{L}$ ) and ketosis (ketonuria or ketonemia) [5]. Whereas euglycemic DKA is a triad comprising of increased anion gap acidosis, evidence of ketosis while being normoglycemic (serum glucose < $250 \mathrm{mg} / \mathrm{dL})$.

The incidence of DKA associated with SGLT- 2 inhibitors has been $\leqslant 0.3 \%$ in the trials involving empagliflozin, dapagliflozin, and ertugliflozin [6-8]. A meta-analysis by Burke et al. looked at 34 cases of DKA caused by SGLT-2 inhibitors of which 25 were of type 2 DM patients [9]. Ertugliflozin was FDA approved in 2017 and is the newest of the SGLT-2 inhibitors. There have been only two reported cases of ertugliflozin-induced 
euglycemic DKA, to our knowledge, thus far per literature review [10-11]. In the Vertis CV trial that looked at the cardiovascular effects of ertugliflozin, 5,490 cases were included. Of which, 19 cases had DKA, seven occurring in the lower dose group (5 mg) and 12 in the higher dose group (15 mg) [8]. Our patient was prescribed a higher dose of $15 \mathrm{mg}$, which is consistent with the notion that higher doses are more prone to the development of eu-DKA.

Most patients developing euglycemic DKA may have a precipitating event. The commonly reported factors include surgery, infections including COVID-19, pericarditis, concurrent ketogenic/low carbohydrate diets, a recent change of diabetic regimen with reduction or omission of insulin dosages, nausea and vomiting, gastroparesis, pregnancy, and acute pancreatitis [12-19]. Diagnosis is often delayed or missed in these settings, like in our case presentation. The reported blood glucose in these cases ranged from 127-212 mg/dL [12-19].

The pathogenesis of eu-DKA is not entirely certain. But, it has been hypothesized that SGLT-2 inhibitors lead to increased glycosuria and reduced serum blood glucose levels, with a shift in metabolism towards the utilization of gluconeogenesis and lipolysis. As there is persistently normal glucose, insulin secretion is reduced to a minimum [2-3]. This phenomenon has the following impact: 1) Increased lipolysis within adipose tissues and production of free fatty acids [20]; 2) Ketone body production in the liver from the transport of fatty acids into the hepatic mitochondria [20]; and 3) SGLT-2 inhibition also stimulates glucagon production, which further induces hepatic production of ketone bodies and resultant ketogenesis [3].

Patients on SGLT-2 inhibitors have reduced glycogen stores and any inciting event can precipitate eu-DKA. Physiologic stressors like surgery and infection can increase stress hormones like cortisol, adrenaline causing further insulin resistance and protein catabolism from alpha and beta receptor stimulation $[15,20]$. Furthermore, SGLT-2 inhibitors can decrease the appetite, as in our patient, and can potentiate the ketogenic state (starvation ketosis).

Typically, from most of the reported cases, eu-DKA has been managed similar to hyperglycemic DKA with intravenous insulin, fluids, and frequent monitoring of serum electrolytes until anion gap acidosis resolution [12-19]. But, patients could be conservatively managed like in our case. Our patient was initiated on lactated Ringer's fluids and a diabetic diet. The AG spontaneously resolved with close monitoring and the patient felt significantly better. This management is particularly possible in type 2 diabetes patients with somewhat reserved endogenous insulin secretory capacity, as with the case management of alcoholic ketoacidosis, a sub-type of euglycemic ketoacidosis. This management strategy, however, would be contraindicated in type 1 diabetes where insulin deficiency is the hallmark of the disease.

\section{Important learning points}

1) The diagnosis of eu-DKA in patients on SGLT-2 inhibitors can be easily missed or delayed, given the nonspecific symptomatology together with normal blood glucose. Therefore, keeping this diagnostic entity in mind is quite critical for early diagnosis and management of this potentially serious adverse event.

2) Higher dose of ertugliflozin is probably more prone to eu-DKA development.

3) Insulin drip is not required in all cases, particularly in type 2 diabetes with partially preserved insulin secretory capacity. Patients can initially be conservatively managed with fluids and initiation of dietary management.

4) With rapidly increasing indications for the use of SGLT-2 inhibitors and increasing usage in heart failure populations, potential acidosis should be considered as it poses a particularly high risk of increased mortality in acute heart failure patients.

\section{Conclusions}

There has been a growing indication for SGLT-2 inhibitors given the evidence from major clinical trials of reduction of cardiovascular events, hospitalization for heart failure, as well as the progression of CKD in both diabetic and nondiabetic populations. Although the incidence of eu-DKA is relatively low, it should be considered especially in patients with risk factors that could precipitate eu-DKA, including those with infection, myocardial infarction, trauma, as well as postoperatively as in our case presentation.

\section{Additional Information \\ Disclosures}

Human subjects: Consent was obtained or waived by all participants in this study. Conflicts of interest: In compliance with the ICMJE uniform disclosure form, all authors declare the following: Payment/services info: All authors have declared that no financial support was received from any organization for the submitted work. Financial relationships: All authors have declared that they have no financial relationships at present or within the previous three years with any organizations that might have an 
interest in the submitted work. Other relationships: All authors have declared that there are no other relationships or activities that could appear to have influenced the submitted work.

\section{References}

1. Horii T, Oikawa Y, Kunisada N, Shimada A, Atsuda K: Real-world risk of hypoglycemia-related hospitalization in Japanese patients with type 2 diabetes using SGLT2 inhibitors: a nationwide cohort study. BMJ Open Diabetes Res Care. 2020, 8:e001856. 10.1136/bmjdrc-2020-001856

2. DeFronzo RA, Norton L, Abdul-Ghani M: Renal, metabolic and cardiovascular considerations of SGLT2 inhibition. Nat Rev Nephrol. 2017, 13:11-26. 10.1038/nrneph.2016.170

3. Bonner C, Kerr-Conte J, Gmyr V, et al.: Inhibition of the glucose transporter SGLT2 with dapagliflozin in pancreatic alpha cells triggers glucagon secretion. Nat Med. 2015, 21:512-7. 10.1038/nm.3828

4. DeFronzo RA, Hompesch M, Kasichayanula S, et al.: Characterization of renal glucose reabsorption in response to dapagliflozin in healthy subjects and subjects with type 2 diabetes. Diabetes Care. 2013, 36:3169-76. 10.2337/dc13-0387

5. Nyenwe EA, Kitabchi AE: The evolution of diabetic ketoacidosis: an update of its etiology, pathogenesis and management. Metabolism. 2016, 65:507-21. 10.1016/j.metabol.2015.12.007

6. Zinman B, Wanner C, Lachin JM, et al.: Empagliflozin, cardiovascular outcomes, and mortality in type 2 diabetes. N Engl J Med. 2015, 373:2117-28. 10.1056/NEJMoa1504720

7. Wiviott SD, Raz I, Bonaca MP, et al.: Dapagliflozin and cardiovascular outcomes in type 2 diabetes . N Engl J Med. 2019, 380:347-5. 10.1056/NEJMoa1812389

8. Cannon CP, Pratley R, Dagogo-Jack S, et al.: Cardiovascular outcomes with ertugliflozin in type 2 diabetes . N Engl J Med. 2020, 383:1425-3. 10.1056/NEJMoa2004967

9. Burke KR, Schumacher CA, Harpe SE: SGLT2 inhibitors: a systematic review of diabetic ketoacidosis and related risk factors in the primary literature. Pharmacotherapy. 2017, 37:187-94. 10.1002/phar.1881

10. Richstein R, Palmeiro C: MON-LB124 euglycemic diabetic ketoacidosis on initiation of ertugliflozin in a patient with type 2 diabetes mellitus precipitated by a ketogenic diet. J Endocr Soc. 2020, 4:MON-LB124. 10.1210/jendso/bvaa046.2286

11. Rameshkumar NS: MON-LB123 diagnosis and management of euglycemic DKA in the setting of SGLT2 inhibitor use and prostate abscess. J Endocr Soc. 2020, 4:MON-LB123. 10.1210/jendso/bvaa046

12. Diaz-Ramos A, Eilbert W, Marquez D: Euglycemic diabetic ketoacidosis associated with sodium-glucose cotransporter-2 inhibitor use: a case report and review of the literature. Int J Emerg Med. 2019, 12:27. 10.1186/s12245-019-0240-0

13. Kameda Y, Kato M, Inoue B, et al.: Euglycemic diabetic ketoacidosis caused by a sodium-glucose cotransporter (SGLT) 2 inhibitor after coronary artery bypass grafting [Article in Japanese]. Kyobu Geka. 2019, 72:354-7.

14. Dass B, Beck A, Holmes C, Morton G: Euglycemic DKA (euDKA) as a presentation of COVID-19. Clin Case Rep. 2020, 9:395-8. 10.1002/ccr3.3540

15. Mendelsohn RA, Taveras AN, Mazer BA, Clayton LM: Euglycemic diabetic ketoacidosis precipitated by SGLT-2 inhibitor use, pericarditis, and fasting: a case report. Clin Pract Cases Emerg Med. 2020, 4:389-92. 10.5811/cpcem.2020.4.46056

16. Steinmetz-Wood S, Gilbert M, Menson K: A case of diabetic ketoacidosis in a patient on an SGLT2 inhibitor and a ketogenic diet: a critical trio not to be missed. Case Rep Endocrinol. 2020, 2020:8832833. 10.1155/2020/8832833

17. Dai Z, Nishihata Y, Kawamatsu N, et al.: Cardiac arrest from acute myocardial infarction complicated with sodium-glucose cotransporter 2 inhibitor-associated ketoacidosis. J Cardiol Cases. 2017, 15:56-60. 10.1016/j.jccase.2016.10.006

18. Madaan M, Aggarwal K, Sharma R, Trivedi SS: Diabetic ketoacidosis occurring with lower blood glucose levels in pregnancy: a report of two cases. J Reprod Med. 2012, 57:452-5.

19. Legaspi R, Narciso P: Euglycemic diabetic ketoacidosis due to gastroparesis, a local experience . J Ark Med Soc. 2015, 112:62-3.

20. Ogawa W, Sakaguchi K: Euglycemic diabetic ketoacidosis induced by SGLT2 inhibitors: possible mechanism and contributing factors. J Diabetes Investig. 2016, 7:135-8. 10.1111/jdi.12401 